Volume 6

\title{
Formation Infırmière au Canada : Enquêtes, Réformes et État-providence
}

Evy Nazon

Université du Québec en Outaouais, evy.nazon@uqo.ca

Follow this and additional works at: https://qane-afi.casn.ca/journal

Part of the Medicine and Health Sciences Commons

\section{Recommended Citation}

Nazon, Evy (2020) "Formation Infirmière au Canada : Enquêtes, Réformes et État-providence," Quality Advancement in Nursing Education - Avancées en formation infirmière: Vol. 6: Iss. 2, Article 9.

DOI: https://doi.org/10.17483/2368-6669.1244

This Article is brought to you for free and open access by Quality Advancement in Nursing Education - Avancées en formation infirmière. It has been accepted for inclusion in Quality Advancement in Nursing Education - Avancées en formation infirmière by an authorized editor of Quality Advancement in Nursing Education - Avancées en formation infirmière. 


\section{Introduction}

Au Canada, la formation des infirmières débute en 1874 lorsque la première école d'infirmières est implantée au General and Marine Hospital à Saint-Catharines en Ontario. Modelée sur les principes établis par Florence Nightingale qui a ouvert la première école d'infirmières à l'Hôpital St-Thomas en 1860, cette école entend améliorer les conditions sanitaires et diminuer les taux de mortalité de la population (Kirkwood, 2005). Par la suite, plusieurs écoles d'infirmières voient le jour à travers le Canada dont celles de l'Hôpital général de Toronto en 1881, l'Hôpital Général de Winnipeg en 1887, l'Hôpital général de Montréal en 1890 et l'Hôpital NotreDame de Montréal en 1898 - première école d'infirmières francophones du Canada - pour ne citer que celles-là (AIIC, 2013). La formation infirmière est alors majoritairement donnée dans les hôpitaux qui s'engagent dans la création d'écoles d'infirmières. Dans ce système d'apprentissage, les étudiantes infirmières reçoivent gratuitement leur formation en échange du travail qu'elles fournissent dans les salles d'hôpitaux (Cohen, 2000). Pour Wytenbroek et Vandenberg (2017), il s'agissait par ce moyen d'apprentissage de rehausser le statut des soins infirmiers et des hôpitaux et d'attirer les patients en mesure de défrayer les coûts reliés à leur hospitalisation. Mais, une telle façon de procéder s'avérait surtout cruciale pour les administrateurs d'hôpitaux, car les étudiantes représentaient une main-d'œuvre peu coûteuse, et le premier but des écoles hôpitaux étant de pourvoir les établissements en main-d'œuvre à bon marché, peu d'importance est accordée aux programmes de formation (Paul et Ross-Kerr, 2011). D'ailleurs, selon Kirkwood (2005), ces écoles n'étaient pas destinées à faire avancer la cause de l'enseignement ni à contribuer à l'avancement des connaissances infirmières, mais à améliorer et optimiser l'organisation hospitalière et la qualité des services aux patients.

Depuis plusieurs années, nombre d'historiens ont réalisé des études sur les balbutiements et l'évolution de la formation des infirmières. Les aspirations éducationnelles ayant conduit à la création des premières écoles d'infirmières, la reconnaissance de leur statut professionnel et l'importance de fournir aux médecins des aides bien formées sont les arguments couramment évoqués pour expliquer le développement des programmes de formation (Allemang, 1974; Bramadat et Chalmers, 1989; Innis, 1970; Kirkwood, 2005; Richardson, 1996; Wood, 1984). De même, des études féministes ont repris ces analyses en y ajoutant des réflexions sur les conditions d'apprentissage et de travail ainsi que l'impact du genre dans le milieu des soins infirmiers. Selon cette nouvelle sensibilité, il fallait remettre en question une histoire qui a construit un récit ignorant le rôle de la femme dans les soins infirmiers (Cohen, 2008; Malka, 2007). Dans cet article, nous voulons nous attarder sur la formation infirmière en prenant en considération le cadre de l'Étatprovidence, c'est-à-dire, une conception de gouvernement dans lequel l'État, par l'établissement d'institutions sociales, joue un rôle important dans la protection et la promotion du bien-être économique et social des individus (Noël, 1996). Un tel cadre peut, nous semble-t-il, apporter des éléments pertinents tant sur le plan politique que social permettant de mieux comprendre l'évolution de la formation infirmière. S'il faut convenir qu'en histoire des soins infirmiers, quelques études se sont penchées sur les politiques sociales mises en place par les États-providence et leurs impacts sur les soins infirmiers, sur la formation et la professionnalisation des infirmières et le rôle de ces dernières dans la normalisation et le gouvernement des populations (Evertsson et Lindqvist, 2005; Gunn, Muntaner, Villeneuve, Chung et Gea-Sanchez, 2019; Nazon 2017; Rafferty, 1996; Rafferty, Robinson et Elkan, 1997), il n'est pas difficile de constater que le cadre de l'État-providence n'est ni structuré par un projet historiographique ni partagé par des questions plus ou moins communes. Du coup, cet article vise à alimenter la discussion en faisant valoir comment la nature politique de la santé a favorisé la mise en place de mesures ayant conduit à des 
réformes d'envergure dans la formation des infirmières au Canada dans les années 1960 permettant ainsi de répondre aux objectifs de l'État-providence.

\section{L'État-providence au Canada}

L'État-providence est un modèle de gouvernement dans lequel l'État joue un rôle clé par la mise en place d'une série de politiques publiques visant l'amélioration, la protection, la promotion de l'état de santé et du bien-être économique et social de la population et la justice sociale. Il connait son apogée entre les années 1960 et 1970, alors que les gouvernements de plusieurs pays occidentaux élargissent les dispositions de protection sociale (Cohen, 2016; Moran, 2000). EspingAndersen (1990) reconnait trois types d'États-providence : libéraux, conservateurs et sociauxdémocrates, mais quel que soit la forme, ce système a pour objectif d'offrir à la population des services sociaux leur permettant de faire face à leurs besoins, notamment ceux liés à la pauvreté, au chômage, au vieillissement, à l'itinérance, à la maladie, à l'immigration, aux blessures au travail et à l'invalidité. Les programmes mis en place visent la réduction des inégalités, l'expansion de la liberté, la répartition équitable des ressources, la promotion de la fraternité et de la solidarité et l'expression de l'action humanitaire. De ce fait, l'État s'implique davantage au niveau social et économique (Vivekanandan et Kurian, 2005).

Si les prémices de l'État-providence remontent au $19^{e}$ siècle, c'est à la fin des années 1920 que des économistes comme Gustav Möller, John Maynard Keynes et William Bedvrige ont favorisé son instauration dans plusieurs pays en déterminant les caractéristiques lui permettant de se développer pendant tout le long du $20^{\mathrm{e}}$ siècle (Vivekanandan et Kurian, 2005). Dans une vision macro-économique, Keynes a présenté une analyse approfondie de ce modèle dans son ouvrage The General Theory of Employment, interest and money publié en 1936. Les idées de Keynes sont reprises dans les recommandations formulées en 1942 par William Beveridge au Royaume-Uni dans son rapport intitulé Report on social insurance and allied services - aussi surnommé Rapport Beveridge - désigné comme le premier document traduisant les grands principes de l'Étatprovidence moderne qui inspire les gouvernements occidentaux à élaborer des réformes visant le bien-être de la population (Merrien, 2007).

Au Canada, les principes de l'État-providence apparaissent en 1935 dans le manifeste publié par la League for Social Reconstruction (LSR) qui prône la répartition équitable des revenus, de la richesse et du pouvoir (Scott, Marsh, Spry, Gordon, Forsey, et Parkinson, 1975). Par la suite, les premiers jalons de l'État-providence sont établis en 1942, lorsque le gouvernement fédéral du Canada charge Leonard C. Marsh de produire un «Plan général de sécurité sociale » calqué sur le modèle du rapport Beveridge (Vivekanandan, 2002). Sorti en 1943, le Rapport Marsh ou Report on social security for Canada présente les méthodes susceptibles de bonifier et d'étendre la portée des lois du domaine de la sécurité sociale et expose les principes pour la mise en place d'un système de sécurité sociale pour le Canada. Plusieurs recommandations du Rapport Marsh sont appliquées à la fin de la Seconde Guerre mondiale et d'autres programmes y découlant, particulièrement dans les domaines de la santé, de l'éducation et des services sociaux, sont introduits de façon effective dans les années 1960 (Horn, 1976) et, ceci s'est fait par l'entremise de professionnels de santé tels que les infirmières. C'est dans ce contexte que les enquêtes sur la formation des infirmières, qui comptent parmi les évènements marquants de la profession infirmière, seront considérées. 


\section{Enquêtes sur la formation infirmière}

Au Canada, dès la fin des années 1920, l'Association des infirmières du Canada (AIC) est préoccupée par les programmes de formation infirmière et croit qu'une réforme s'impose. Selon cette association, la révision des programmes représente le meilleur moyen pour ouvrir de nouveaux horizons à la profession et pour corriger les problèmes professionnels, par exemple, la qualité de l'éducation, l'offre excédentaire d'infirmières diplômées et le chômage des infirmières (AIIC, 2013). Ces préoccupations ont conduit l'AIC à mener des enquêtes visant à étudier l'état de l'enseignement infirmier au Canada. Dans les lignes qui suivent, nous allons nous attarder sur deux principales enquêtes mandatées par l'AIC : le Survey of nursing education in Canada et le Spotlight on nursing education.

\section{Survey of nursing education in Canada (Rapport Weir)}

En juin 1927, les responsables de l'AIC et de l'Association Médicale Canadienne (AMC) décident de financer l'enquête intitulée Survey of Nursing Education in Canada. Dès lors, un comité mixte est créé. Il est composé de six membres: les infirmières Jean Gunn, Katleen Russel et Jean Browne, et les médecins A. T. Bazin, Duncan Graham et G. Stewart Cameron. Ce comité convient qu'une telle étude devait être pilotée par une personne compétente, expérimentée et neutre. À cet effet, le professeur George M. Weir, chef du département d'éducation de l'Université de la Colombie-Britannique, est désigné pour étudier tous les aspects de la profession et de la formation des infirmières (The Canadian Nurse, 1932). L'enquête est menée de 1929 à 1931 et le rapport - Rapport Weir - est publié en 1932. Les données sont recueillies dans 65\% des écoles d'hôpitaux à travers tout le Canada (Godbout, 1933) et, à l'instar du Goldmark Report publié en 1923 aux États-Unis, le Rapport Weir trace un portrait peu flatteur des écoles d'infirmières au Canada:

L'Église, au cours des premiers siècles de l'ère chrétienne et au Moyen âge, a exercé une forte influence sur toutes les manifestations de la vie professionnelle. La loi et la médecine se sont dégagées d'un pareil contrôle. Il n'en est pas ainsi de la profession des institutrices, ni de celle des gardes-malades. Dans plusieurs centres du Canada, un groupe important de gardes-malades professionnelles suit les traditions ecclésiastiques [...]; au point de vue spirituel cet état ne peut être des plus désirables [...]. Cependant, il n'en reste pas moins que cette tradition, qui date du temps des missionnaires dans ce pays [...] a exercé et exerce encore au point de vue économique une influence qui peut être considérée comme quelque peu préjudiciable à l'évolution de la profession infirmière (Weir, 1932, p. 27) [Traduction libre].

De plus, Weir dénonce le temps précieux que les infirmières perdent à accomplir diverses tâches ménagères: «Elles passent plus de 75\% de leur temps à balayer, polir, épousseter, nettoyer et désinfecter les murs, meubles, matelas et oreillers. »(Weir, 1932, p.191) [Traduction libre]. L'enquête fait également ressortir que même si les écoles d'infirmières en milieu hospitalier se sont multipliées par sept pour la période allant de 1913 à 1930, aucune d'entre elles n'a pu fournir un enseignement adéquat permettant la formation d'infirmières parfaitement qualifiées ni au niveau théorique ni dans le domaine pratique qui les préparerait à répondre à toutes les tâches qu'elles sont appelées à remplir après l'obtention de leur diplôme :

L'une des causes principales de l'impasse dans laquelle se trouve, au Canada, la profession d'infirmière du point de vue économique et pédagogique, est que, dans les diverses provinces, cette profession s'est développée sans coordination quant aux buts à atteindre et 
sans compréhension des besoins sanitaires et des intérêts économiques de la société. [...]. Si la profession d'infirmière a tenu de moins en moins compte des problèmes du public, c'est surtout parce qu'elle n'était pas en possession des moyens scientifiques coordonnés pour analyser et déterminer ses besoins (Gunn, 1934, p. 603).

Le rapport Weir suggère que les écoles d'infirmières soient incorporées dans le système éducatif général du pays et subventionnées par des fonds publics (Wood, 1984). Il souligne également l'importance d'améliorer les programmes de formation et recommande d'établir un niveau uniforme d'enseignement dans toutes les écoles d'infirmières au Canada; de créer des écoles de formation universitaire pour les infirmières qui octroieraient des diplômes de baccalauréat; et de mettre en place un conseil des infirmières qui aurait pour mission de surveiller la formation des infirmières et de procéder à des recherches sur l'état des soins infirmiers au Canada (Weir, 1932). À cet effet, il convient de souligner que certaines de ces recommandations avaient déjà été émises depuis 1914 par le Special Committee in Nursing Education dirigé par les professeurs Falconer de l'Université de Toronto et McLean de l'Université du Manitoba qui avaient notamment réclamé une scission entre les écoles de formation infirmière et les hôpitaux. Mais, une telle demande formulée dans les débuts de la Grande Guerre n'avait pas retenu l'attention des instances concernées (Innis, 1970).

Bien que les recommandations du Rapport Weir n'aient pas conduit à une révolution de la formation des infirmières, des mesures ont été prises par les associations tant nationale que provinciales afin d'en implanter quelques-unes, dont la création de comités pour la refonte des programmes et curricula, l'amélioration des conditions de vie et des heures de travail des étudiantes et des infirmières, ainsi que la publication d'un guide de programmes d'études pour inciter les écoles hôpitaux à atteindre plus d'autonomie éducative (Bramadat et Chalmers, 1989; The Canadian Nurse, 1932). Toutefois, les déboires engendrés par la crise économique des années 1930, la forte prépondérance de la religion dans le domaine de la santé et de l'éducation et le peu d'engagement de l'État, le rôle influent des administrateurs d'hôpitaux et la multiplication des postes d'infirmières dans les hôpitaux après la Seconde Guerre mondiale ont contribué à garder la formation infirmière dans les hôpitaux, un modèle qui perdurera jusque dans les années 1970 (CASN, 2012).

La publication du Rapport Weir correspond au début de l'État-providence au Canada, mais il n'en demeure pas moins que les recommandations émises par la League for Social Reconstruction (LSR) en 1935 reconnaissaient déjà les soins de santé tels que la santé publique, le contrôle des maladies, l'accès aux soins et au personnel de santé comme étant des services sociaux de base qui devraient être fournis à toute la population (Horn, 1976).

\section{Helen Mussallem et l'enquête Spotlight on nursing education}

Après l'enquête menée par Weir, l'AIC commande, quelques années plus tard, une autre étude sur la formation des infirmières: le Spotlight on nursing education. L'AIC estimait, d'une part, que la possibilité d'évaluer ses propres programmes de formation au moyen d'un processus d'agrément représentait un élément important dans l'amélioration de l'éducation des infirmières et, d'autre part, il devenait essentiel d'obtenir du financement et la volonté politique pour instaurer un changement véritable, car jusqu'alors, l'absence d'un plan bien élaboré et le manque de financement avaient anéanti les efforts déployés pour bonifier les programmes de formation (AIIC, 2013). Ainsi, au début des années 1950, la volonté d'aller vers une formation renouvelée des infirmières avait ré-émergé et cette amélioration devait, sans conteste, passer par l'indépendance 
de la formation, c'est-à-dire, par l'abolition des écoles-hôpitaux telle que stipulée dans le Rapport Weir. En 1957, l'AIC réitère sa volonté de mettre en place un processus permettant d'atteindre cet objectif et la première étape consiste à l'évaluation et l'agrément des programmes de formation des écoles d'infirmières. Avant de lancer ce processus au niveau national, l'AIC décide d'entreprendre une étude pilote, The pilot project for evaluation of schools of nursing, dont l'objectif est d'évaluer dans quelle mesure les écoles étaient préparées à un système national d'agrément (AIIC, 2013).

Vingt-cinq écoles d'infirmières au Canada, dont au moins une dans chaque province, sont sélectionnées pour faire partie de l'enquête. La responsable de ce projet est Helen K. Mussallem, infirmière chevronnée et directrice de l'Hôpital général de Vancouver. Défini comme la reconnaissance officielle et publique des programmes d'une institution de formation après une évaluation crédible, l'agrément vise à assurer que la formation est fondée sur des normes reconnues scientifiquement (Mussallem, 1960a) et représente un moyen de stimuler les écoles infirmières vers une amélioration constante de leurs programmes de formation (Lefebvre, 1960). Au cours de l'enquête, Mussallem visite les écoles sélectionnées et mène des entrevues auprès de 2000 infirmières, administrateurs et éducateurs qui sont en lien avec les systèmes de santé et d'éducation. Le but est de clarifier les programmes en soins infirmiers, valider la formation dans les écoles et aider les enseignants à reconnaitre et développer leurs compétences pour trouver des solutions aux problèmes identifiés (Mussallem, 1960a). Bien que mitigées, les réactions par rapport à l'enquête demeurent en général positives comme en témoigne la directrice d'une des écoles visitées:

Le programme d'évaluation et d'agrément des écoles est un moyen de les aider à relever leurs standards. Ce n'est ni une perte de temps ni une perte d'argent. Au contraire, il permet aux écoles d'infirmières de suivre le rythme du progrès. Ayant participé au Projet Pilote, notre vision s'est considérablement modifiée. Nous sommes plus conscients des points à améliorer. Les gens font un meilleur travail quand ils savent ce qu'ils doivent faire, quand ils comprennent ce qui constitue un travail bien fait en termes de résultats scientifiques, et quand ils savent que ce qu'ils font a de la valeur (Mussallem, 1960a, p. 233).

Les données obtenues auprès des écoles ont révélé de nombreux sujets de préoccupation. Par exemple, 30\% des enseignants n'étaient nullement préparés à l'enseignement; 48,8\% d'entre elles avaient un certificat ou un diplôme obtenu après une année d'études dans une université alors que $21,5 \%$ avaient un baccalauréat et 3,8\% avaient une maîtrise. En ce qui concerne les étudiantes, les résultats ont montré que dans la majorité des écoles, elles étaient considérées comme des membres actifs du personnel et leurs heures étaient prévues en conséquence. Dans la plupart des cas, les étudiantes travaillaient dans les milieux cliniques quatre à huit heures de plus par semaine que les infirmières diplômées et assumaient des tâches de soins et administratives au-delà de leur niveau de préparation et compétence (Mussallem, 1960a). Mussallem indique :

Je n'avais aucune idée que les endroits qui se proclamaient écoles de soins infirmiers pratiquaient, disons, l'esclavage. Ils se contentaient d'exploiter les élèves, s'en servant comme personnel pour l'hôpital. On se souciait si peu de leur formation et on les logeait dans les pires conditions. Un peu moins dans l'Ouest, mais dans les provinces de l'Atlantique ou dans des petites villes du Québec, cela dépassait presque l'entendement. [...]. Des gens qui disaient qu'ils faisaient de la formation alors qu'ils mettaient les élèves infirmières dans un service pour qu'elles glanent des miettes de connaissances comme elles le pouvaient. C'était si rudimentaire, mon stylo crachait des flammes pendant que 
j'écrivais. Je n'en revenais pas. [...]. Rétrospectivement, c'était vraiment majeur, car nous pourrions encore nous débattre dans ces horribles écoles hospitalières. J'ai été persécutée par l'Association des hôpitaux, qui ne voulaient pas perdre leurs écoles. Je suis allée à une de leurs réunions, auxquelles ils m'invitaient toujours, et à cette réunion, ils ont parlé de cette femme qui colportait toutes ces histoires sur les terribles écoles hospitalières et dit qu'elle devrait être remise à sa place. Ils ne savaient même pas que j'étais assise au fond de la salle. Certains de ces administrateurs m'en ont voulu pendant des années (The Canadian Nurse, 2013, p. 4) [Traduction libre].

Mussallem (1960b) souligne que les normes d'éducation étaient honteuses et que les infirmières n'étaient rien de plus que des travailleuses sous contrat qui avaient grandement besoin d'être libérées de leurs conditions de travail. La plupart des hôpitaux, note-t-elle, ont été responsables de la formation des infirmières et les ont forcées à vivre sur place, se garantissant ainsi une main-d'œuvre disponible à bon marché. De telles constatations rejoignent celles formulées par le professeur Weir en 1932 soulignant ainsi le peu de changements dans les conditions de formation et de travail des infirmières.

À la suite du projet pilote, Mussallem rédige en 1960 un rapport intitulé Spotlight on nursing education dont les recommandations principales sont: 1) de procéder à un réexamen et une étude de l'ensemble de la formation infirmière au Canada; 2) de lancer un programme d'amélioration de la formation dans les écoles d'infirmières; 3) d'élaborer un programme afin d'évaluer la qualité des services infirmiers dans les milieux où les étudiants font leur stage clinique; et 4) d'instaurer un programme d'agrément des écoles infirmières (Mussallem, 1960b). Mussallem prend l'exemple de la formation des infirmières aux États-Unis qui avec deux catégories d'infirmières, celles préparées dans les universités et celles formées dans les collèges, parviennent à développer chez les étudiantes les connaissances nécessaires pour répondre adéquatement au besoin de la population en termes de soins infirmiers globaux (Mussallem, Articles about, 19781985). La formation universitaire est largement discutée tant au niveau de l'AIC que des organisations provinciales et, selon ces différentes instances, il est impératif qu'elle devienne une réalité à l'échelle nationale, car même si elle a débuté en 1919 à l'Université de la ColombieBritannique, selon Mussallem, cette formation n'a pas eu l'impact qu'elle devrait ou pourrait avoir sur les soins de santé au Canada. La raison, souligne-t-elle, est, malheureusement évidente, ces programmes n'ont pas produit des praticiens qui ont pu avoir l'opportunité de pratiquer des soins infirmiers d'une qualité supérieure à ceux des infirmières diplômées des écoles d'hôpitaux (Mussallem, Articles about, 1978-1985). Bien que les résultats de l'enquête se soient révélés décevants, car au moins $84 \%$ des écoles, soit 21 sur 25 , ne satisfaisaient pas aux critères d'agrément, ceux-ci ont permis la mise en place d'un programme d'amélioration des écoles afin d'en augmenter la qualité (AIIC, 2013).

Le rapport de Mussallem est publié durant ce que certains auteurs reconnaissent comme la phase d'expansion et interventionniste de l'État-providence où le rôle accru de l'État dans les secteurs économiques et sociaux devient plus évident avec l'introduction des mesures de protection sociale et l'accessibilité à l'enseignement supérieur et des soins de santé à une plus grande partie de la population (Merrien, 2007). D'ailleurs, les réformes d'envergure au niveau de la formation des infirmières ont débuté au cours des années 1960 lorsque la Commission royale d'enquête sur les services de santé a appuyé plusieurs des recommandations formulées par l'AIIC et orienté la formation des infirmières vers les collèges et universités placés sous l'égide du ministère de l'Éducation (AIIC, 2013). En fait, la formation renouvelée réclamée par l'AIC depuis 
le Rapport Weir en 1932 et reprise dans les années 1960 rejoint un ordre du discours plus large, celui de l'État-providence à la lumière duquel il nous est possible de mieux comprendre le pouvoir des décisions sanitaires et politiques sur l'éducation des infirmières et des réformes qui y sont instaurées donnant un aperçu de la politique complexe de la santé et des soins de santé.

\section{L'État-providence et les soins infirmiers au Canada}

Les politiques de santé prônées par l'État-providence exigent un personnel spécialisé et les infirmières, en tant que principales prestataires de soins, ont été d'une grande utilité sociale pour la rationalisation systémique de l'offre de soins envisagée par l'État-providence (Evertsson et Lindqvist, 2005; Wrede, 2010). Au Canada, l'État-providence s'est impliqué de façon importante dans les programmes d'éducation des infirmières. Celles-ci occupent en effet un rôle de premier plan, d'une part, par leur savoir et, d'autre part, par l'enseignement qu'elles fournissent aux individus et l'influence qu'elles exercent auprès de la population (Nazon, 2017). Des réformes relatives à la formation des infirmières ont été mises en place à la suite du rapport de la Commission royale d'enquête sur les services de santé ou Commission Hall conduite par le juge Emmett M. Hall, Wallace McCutcheon, Alice Girard, infirmière et professeure à l'Université de Montréal et les médecins : David M. Baltzan, C. L. Strachan, Arthur F. Van Wart et le professeur O. J. Firestone. Cette commission avait pour but d'enquêter sur les services sanitaires existants, d'inventorier les ressources disponibles et d'identifier les besoins en soins de santé de la population canadienne afin de lui fournir les meilleurs soins de santé possibles (Gouvernement du Canada, 2005). Du reste, il importe de souligner que l'étude de Mussallem, Nursing Education in Canada, et un mémoire préparé par l'AIC où sont formulées 25 recommandations pour améliorer les services de soins infirmiers au Canada figurent parmi les études spéciales retenues dans l'élaboration du rapport de la Commission Hall (AIIC, 2013).

Publié en 1964, ce rapport recommande l'élaboration d'une politique nationale et un programme de soins de santé complet dans les domaines suivants : 1) services de santé; 2) effectifs, accommodations et recherche en santé; et, 3) financement du système de santé. Tout comme le Rapport Weir en 1932, il démontre des insuffisances notoires dans la formation infirmière et, afin d'y remédier, propose plusieurs recommandations dont: la formation d'une infirmière sur trois au niveau universitaire, la mise sur pied de programmes de baccalauréat en sciences infirmières dans les universités canadiennes et la création d'un programme de maîtrise en sciences infirmières (Naegele, 1964).

La Commission Hall a également recommandé qu'il y ait deux catégories d'infirmières, celles formées dans un programme universitaire (environ 20\%) pour occuper des postes d'administrateur, d'instructeur et de supervision, et celles formées comme infirmière de chevet dans un programme qui octroierait un diplôme après deux ans d'étude (The Canadian Nurse, 1964). De surcroît, le rapport fait valoir que la formation des infirmières devrait être organisée et financée comme les autres formations professionnelles et, dans les articles 124 à 126, il est demandé que, dans chaque province, un comité spécial soit chargé d'établir le programme de formation des infirmières; que les budgets des écoles d'infirmières soient séparés de ceux du service infirmier de l'hôpital afin que celles-ci servent uniquement à la formation des élèves; que les hôpitaux mettent leurs installations éducatives et cliniques à la disposition des élèves infirmières pour que ces dernières acquièrent des connaissances et de l'expérience clinique sans avoir à faire du service infirmier (Nazon, 2017). 
Plusieurs reconnaissent les avantages de ces réformes, car ce qui suffisait en matière d'éducation infirmière n'est plus valable et il est nécessaire de fournir aux infirmières une formation qui leur permette d'être à la hauteur de leur profession. Mussallem (1961) soutient que compte tenu des développements scientifiques et sociologiques, les besoins en santé de la société ont changé et, pour cela, les soins infirmiers et la formation des infirmières doivent également changer pour répondre aux nouveaux besoins. Les mérites de la formation universitaire ont été vantés par d'autres acteurs concernés par la formation des infirmières. Par exemple, pour Margaret McLean du Département national de la santé et du bien-être, il est important d'aider les infirmières à devenir des praticiennes qualifiées par le biais de programmes universitaires de cycles supérieurs (McLean, 1964). De son côté, Claire Gagnon (1967), directrice des services infirmiers, Assurancehospitalisation du Ministère de la Santé du Québec remet en question la formation qui était donnée aux infirmières dans les écoles d'hôpitaux. Elle note que l'éducation des infirmières est sur la « voie du progrès » et que « les soins infirmiers ont cessé d'être purement un art transmis selon un mode artisanal ». Elle soutient l'idée que c'est le milieu académique (ex. : universités) qui doit désormais être garant de la formation scientifique infirmière.

Responsable du devenir et du bien-être de la société, l’État-providence articule à la fois la santé, l'éducation, l'économie et le social en se dotant d'institutions, de pratiques et de ressources lui permettant d'assurer la protection sociale, la promotion de l'état de santé et le bien-être économique de la population.

\section{Discussion}

Plusieurs écrits en histoire des soins infirmiers montrent que l'objectif des infirmières a toujours été de prodiguer des soins qui favorisent le bien-être et la santé - santé étant comprise le plus souvent comme exempte de maladie - des patients (Keeling, Hehman, et Kirchgessner, 2017; D’Antonio et Lewenson, 2010). Par ailleurs, Rafferty (1996) note que les historiens tendent à prioriser le rôle joué par les leaders infirmières dans la réalisation des réformes de l'éducation et des soins infirmiers et à sous-estimer le rôle de l'État alors qu'une analyse fine des politiques sociales en Grande-Bretagne révèle les efforts considérables déployés par l'État-providence pour organiser et financer la santé, l'éducation des infirmières afin que celles-ci soient en mesure de répondre aux objectifs du système de santé.

Ainsi, au-delà de la conception bienfaisante et curative de la santé et du rôle des leaders dans la formation infirmière, il convient de considérer que la santé est politique. Selon Bambra, Fox, et Scott-Samuel (2005), la santé, la promotion de la santé et la prévention de la maladie sont profondément politiques. Ils avancent plusieurs raisons pour expliquer ce lien : 1) la santé étant liée aux déterminants sociaux, elle dépend de ce fait des interventions politiques ; 2) le droit à la santé, à un niveau de vie adéquat et au bien-être fait partie des droits humains et représente un aspect de la citoyenneté ; enfin, 3) la santé doit être considérée dans le cadre d'un système économique, social et politique où il y a exercice de pouvoir, car lorsqu'il faut apporter des changements au système de santé, une prise de conscience politique et une lutte politique sont nécessaires. Dans la même veine, Nunes (2012) soutient que la santé ne peut être isolée de la sphère politique, car toutes les réponses aux problèmes de santé dépendent des décisions politiques (ex. : allocation des ressources, promotion de la santé, etc.). Il souligne que plus qu'une condition médicale ou un ensemble de solutions techniques, la santé doit être comprise comme un ensemble de perceptions et de pratiques qui mobilisent des formes de pouvoir qui se rattachent à des contextes sociaux et politiques et, dans le cas qui nous concerne ici, à l'État-providence. Pour Foucault (1994), l'apparition de la santé comme objet politique s'explique grâce au processus qui 
fait du bien-être un des objectifs essentiels du pouvoir politique et qui s'obtient par des jeux subtils d'interventions et de libertés déterminés selon un savoir spécifique. Dans le faisceau des services, la santé et l'éducation représentent alors des secteurs d'intervention majeure des États-providence.

Plus encore, la santé représente un pilier important dans la construction de l'Étatprovidence. Dans de nombreux pays, plusieurs éléments mettent en évidence l'importance des soins de santé. Les améliorations de la médecine curative (ex. : traitement, nouvelles méthodes chirurgicales), les innovations sociales (ex.: rationalisation du travail, industrialisation), les avancées dans le domaine des germes et des microbes et la transformation des hôpitaux en institutions de pratiques scientifiques démontrent l'efficacité thérapeutique des soins de santé (Moran, 2000). La plupart des États-providence vont établir des politiques de santé qui garantissent l'accès universel aux soins médicaux (assurance-maladie) et des dispositions en matière de santé publique (hygiène, prévention des maladies, etc.) afin d'assurer la sécurité et le bien-être des individus et ainsi contribuer à la gestion politique des populations (Jordan, 2010). D’abord perçue comme une charge pour la nation, la santé devient une ressource et un bien social qui vise à garantir l'efficacité et la productivité de la population comme force de travail (Therborn, 2011; Ulmann, 2009).

De même, l'éducation fait partie intégrante des politiques sociales élaborées par l'Étatprovidence (Crook, 2007). L'expansion du système éducatif et particulièrement de l'enseignement supérieur et la nécessité d'une main-d'œuvre mieux scolarisée représentent un moyen d'amélioration du statut socio-économique des individus et de réduction des risques sociaux pour la population (Allmendinger et Leibfried, 2003; Busemeyer et Nikolai, 2010). Le niveau de scolarité et le marché du travail étant étroitement liés, un faible niveau de scolarité peut facilement conduire à des positions précaires sur le marché du travail et à des problèmes sociaux comme la pauvreté, le chômage ou la maladie (Hega et Hokenmaier, 2002) et, avec les visées de l'État providence, il faut à tout prix miser sur l'éducation afin de se prémunir contre les différents risques et bénéficier de conditions de vie minimales (Di Stasio et Solga, 2017). À cet égard, l'éducation, la légitimité et les connaissances scientifiques deviennent également des ressources importantes pour l'État-providence. On comprend alors l'importance de la formation des infirmières pour le système de santé et pour l'État-providence.

En 1961, Mussallem écrivait: "Pourquoi la voie du changement dans la formation infirmière a-t-elle été si difficile? Peut-être que le problème est que nous n'avons jamais trouvé le bon chemin. » (p.530) [Traduction libre]. Il semble en effet que les infirmières jusque-là n'avaient pas trouvé le bon chemin pour apporter des changements significatifs au niveau de leur formation. Il appert que plusieurs des recommandations formulées par Weir et Mussallem n'ont pas été réellement prises en considération par les instances politiques. Il a fallu celles émises par la Commission Hall sous l'égide de l'État-providence pour voir d'importantes réformes être implantées rapidement au niveau de l'éducation des infirmières (CASN, 2012). Tout d'abord, ces recommandations ont permis la séparation de la formation infirmière du service hospitalier dans plusieurs provinces canadiennes. En effet, dans les années 1960-1970, la formation infirmière dans plusieurs hôpitaux du Québec, de l'Ontario, de la Saskatchewan et de l'Alberta a été délaissée et les collèges communautaires - Collèges d'enseignement général et professionnel (CÉGEP) au Québec - sont devenus majoritairement la norme. En 1978, l'AIC a dénombré 104 programmes de formation préparant les infirmières au diplôme et, de ce nombre, 73 d'entre eux étaient dispensés par les collèges communautaires ou cégeps, 6 par des écoles d'infirmières indépendantes ou régionales et 25 seulement par des écoles liées à des hôpitaux (AIIC, 1981). De plus, de 
nouvelles écoles de sciences infirmières ont été créées dans les universités à travers tout le Canada, par exemple, au Memorial University, à l'Université Laval, à l'Université de Moncton et à l'Université de Saskatchewan (Paul et Ross-Kerr, 2011). De même, au cours de ces mêmes années, les programmes de maîtrise en sciences infirmières se sont multipliés. Après le premier programme démarré avec le support de la Fondation Kellogg à l'Université Western Ontario en 1959, plusieurs autres universités en Alberta, au Québec, en Colombie-Britannique ont aussi développé des programmes de maîtrise en sciences infirmières (CASN, 2012). Au regard des objectifs de l'Étatprovidence, il fallait une éducation infirmière plus poussée et un personnel spécialisé pour garantir l'efficacité du système de santé. Et, bien que les facteurs ayant provoqué ces changements soient nombreux: apport de l'AIIC, contribution d'infirmières comme Mussallem, progrès technologiques, etc., l'État-providence représente un élément important ayant favorisé la transformation de l'éducation des infirmières au Canada.

\section{Implications}

Faisant partie du système de santé, les infirmières ont un lien étroit avec les objectifs de santé et d'éducation de l'État-providence. Leur rôle est important dans l'administration et la mise en œuvre des stratégies déterminées par l'État. Elles servent de lien direct entre les services fournis par l'État-providence et les citoyens, en particulier, en ce qui concerne la promotion de la santé, la prévention des maladies, le respect des mesures d'hygiène et les droits sociaux. Les soins infirmiers étant intimement liés aux politiques de l'État-providence, il importe d'approfondir les analyses historiques sur l'aspect politique de la santé et ses impacts sur la formation des infirmières. De nombreux aspects de l'État providence dans l'histoire des soins infirmiers demeurent inexploités et justifieraient un examen attentif qui permettrait de mettre en évidence la façon dont la profession infirmière a interagi avec les structures et les objectifs de l'État providence. D'autres recherches en histoire des soins infirmiers pourraient se pencher sur les politiques liées à la santé, aux inégalités en santé et au rôle des infirmières étant donné les effets de l'État-providence sur le marché du travail, le revenu et les inégalités sociales au sein de la population. Des recherches pourraient également inclure les effets des nouvelles formes d'organisation des soins de santé sur la demande d'infirmières après les réformes de l'Étatprovidence, leurs responsabilités et leurs rôles dans les hôpitaux et dans les nouveaux milieux de soins communautaires. L'État-providence a été marquant pour les soins infirmiers et il est important que les historiens spécialistes de cette question continuent de réfléchir sur le rôle stratégique des infirmières en reliant soins infirmiers, éducation, santé et politique.

\section{Conclusion}

L'essor de l'État-providence dans les années 1960 marque un tournant majeur dans l'histoire des soins infirmiers au Canada et, particulièrement, de l'histoire de la formation des infirmières. Malgré les enquêtes menées par les leaders infirmières sur l'état de l'éducation des infirmières et les nombreuses recommandations qu'elles ont formulées, c'est au travers des réformes instituées par l'État-providence dans le système de santé à la suite des recommandations émises par la Commission royale d'enquête sur les services de santé que des changements en profondeur ont été instaurés au niveau des écoles et des programmes en soins infirmiers dont le plus remarquable a été le passage de la formation du milieu hospitalier à l'enseignement dans les collèges et plus tard, dans les universités. La santé étant hautement politique, il est important que les analyses historiques s'approprient une telle définition de la santé et présentent de façon plus critique les objectifs de l'État-providence et ses implications sur la profession infirmière afin de montrer le rôle stratégique des infirmières au sein des systèmes de santé. 


\section{Références}

Allemang, M. M. (1974). Nursing education in the United States and Canada, 1873-1950: Leading figures, forces, views on education (Thèse de doctorat inédite). University of Washington, Washington.

Allmendinger, J., et Leibfried. S. (2003). Education and the welfare state: the four worlds of competence production. Journal of European Social Policy, 13(1): 63-81.

Association des infirmières et infirmiers du Canada [AIIC]. (2013). Cent ans de service. Association des infirmières et infirmiers du Canada. https://www.cna-aiic.ca/$/$ media/cna/page-content/pdffr/cna_history_book_f.pdf?la=fr\&hash=C5EEAF21DD014555412B16642872A0739780 D9F3

Association des infirmières et infirmiers du Canada [AIIC]. (1981). La septième décennie 19691980. Association des infirmières et infirmiers du Canada. https://www.cna-aiic.ca//media/cna/page-content/pdffr/the seventh_decade_f.pdf?la=fr\&hash=EBE03E42180EC97A9EC5725328BBFCC30 D1CBDA2

Bambra, C., Fox, D., et Scott-Samuel, A. (2005). Towards a politics of health. Health Promotion International, 20(2), 187-193.

Bramadat, I. J., et Chalmers, K. I. (1989). Nursing education in Canada: historical progress contemporary issues. Journal of Advanced Nursing, 14(9), 719-726.

Busemeyer, M. R., et Nikolai, R. (2010). «Education ». Dans F.G. Castles, S. Leibfried, J. Lewis, H. Obinger, et C. Pierson (Eds), The Oxford Handbook of the Welfare State (pp. 494-508). Oxford University Press.

Canadian Association of Schools of Nursing [CASN]. (2012). Ties that bind: the evolution of education for professional nursing in Canada from the $17^{\text {th }}$ to the $21^{\text {st }}$ century. Ottawa, ON: Canadian Association of Schools of Nursing. https://www.casn.ca/wpcontent/uploads/2016/12/History.pdf

Cohen, S. S. (2016). A primer on political philosophy. Dans D.J. Mason, J.K. Leavitt, et M.W. Shaffee, Policy \& politics in nursing and health care (7th Ed.) (chap. 6). Elsevier.

Cohen, Y. (2000). Profession infirmière : Une histoire de soins dans les hôpitaux du Québec. Les Presses de l'Université de Montréal.

Cohen, Y. (2008). Réflexions sur l'histoire des infirmières au Québec. Recherche en Soins Infirmiers, 2(93), 84-93.

Crook, D. (2007) Education, Health and Social Welfare. History of Education, 36(6), 651-657. doi:10.1080/00467600701619630

D’Antonio, P., et Lewenson, S. (2010). Nursing Interventions Through Time: History as Evidence. Springer Publishing Company.

Di Stasio, V., et Solga, H. (2017). Education as social policy: An introduction. Journal of European Social Policy, 27(4) 313 -319. doi:10.1177/0958928717728712journals.sagepub.com/hom 
Esping-Andersen, G. (1990). The Three Worlds of Welfare Capitalism. Princeton University Press.

Evertsson L., et Lindqvist R. (2005). Welfare state and women's work: the professional projects of nurses and occupational therapists in Sweden. Nursing Inquiry, 12(4), 256-268.

Features of the report of the Survey of Nursing Education in Canada. (1932). The Canadian Nurse, 28(3), 127-131.

Foucault, M. (1994). « The birth of social medicine ». Dans P. Rabinow et N. Rose, The essential Foucault: selections from essential works of Foucault, 1954-1984 (pp. 319-337).The New Press.

Gagnon, C. (1967). Correspondance. L'hôpital et l'enseignement infirmier - communication présentée au congrès de l'Association des Hôpitaux de la Province de Québec, 02 mai 1967. Fonds d'archives de l'école d'infirmières de l'Hôpital Notre-Dame de Montréal (Boite A-167 C). Archives de l'Hôpital Notre-Dame de Montréal, Montréal, Canada.

Godbout, C. (1933). Résumé de l'enquête du Dr. G. M. Weir au sujet de l'état du Nursing au Canada. La Garde-Malade Canadienne-Française, 6(1), 23-25.

Gouvernement du Canada. (2005). Commission royale d'enquête sur les services de santé, 1961 1964. https://www.canada.ca/fr/sante-canada/services/systeme-soins-sante/commissionsenquetes/commissions-federales-soins-sante/commission-royale-services-sante.html

Gunn, J. I. (1934). Enquête sur la formation au Canada. La Garde-Malade CanadienneFrançaise, 7(10), 600-611.

Gunn, V., Muntaner, C., Villeneuve, M., Chung, H., et Gea-Sanchez, M. (2019). Nursing professionalization and welfare state policies: A critical review of structural factors influencing the development of nursing and the nursing workforce. Nursing Inquiry, 26(1): e12263. doi:10.1111/nin.12263.

Hega, G. M., et Hokenmaier, K. G. (2002). The welfare state and education: a comparison of social and educational policy in advanced industrial societies. German Policy Studies, 2(1), 1-29:

Helen Mussallem: in her own words. (2013). The Canadian Nurse, 109(2), 22-25. https://www.canadian-nurse.com/en/articles/issues/2013/february-2013/helen-mussallemin-her-own-words

Horn, M. (1976). Leonard Marsh and the coming of a Welfare state in Canada: a review article. Histoire Sociale/Social History, 9(17), 197-204.

Innis, M. A. (1970). Education in a changing society. University of Toronto Press.

Jordan, J. (2010). Institutional Feedback and Support for the Welfare State: The Case of National Health Care. Comparative Political Studies, 43(7), 862-885. doi:10.1177/0010414010361342

Keeling, A. W., Hehman, M. C., et Kirchgessner, J. C. (2017). History of Professional Nursing in the United States: Toward a Culture of Health. Springer Publishing. 
Kirkwood, L. (2005). Assez mais pas trop : la formation en nursing au Canada anglais (18742000). Dans C. Bates, D. Dodd, et N. Rousseau, Sans Frontières : Quatre siècles de soins infirmiers canadiens (pp.183-196). Les Presses de l'Université d'Ottawa.

Lefebvre, D. (1960). Behind the scenes on accreditation. The Canadian Nurse, 56(10), 893-898.

Malka, D. G. (2007). Daring to Care: American Nursing and Second-wave Feminism. University of Illinois Press.

McLean, M. (1964). Improvement of nursing practice. The Canadian Nurse, 60(10), 955-960

Merrien, F.-X. (2007). L'État-providence. Presses Universitaires de France.

Moran, M. (2000). Understanding the welfare state: the case of health care. British Journal of Politics and International Relations, 2(2), 135-160.

Mussallem, K. (1960a). A Review of the Pilot project. The Canadian Nurse, 56(3), 231-234.

Mussallem, K. (1960b). Spotlight on nursing education. The Canadian Nurse, 56(10), 901-906

Mussallem, K. (1961). A whispering into the wind. The Canadian Nurse, 57(6), 529-531.

Mussallem, K. [?]Articles about Mussallem, Helen K., 1978-1985. Fonds Helen K. Mussallem (R8293, Boîte 28, volume 10 Dossier 4). Bibliothèque et Archives Canada, Ottawa, Ontario.

Naegele, K. (1964). Study of nursing education. The Canadian Nurse, 60(9), 865-869.

Newfoundland rendezvous. (1964). The Canadian Nurse, 60(9), 845-858.

Nazon, E. (2017). Analyse de discours de la construction du savoir infirmier au Québec, 18981970 (Thèse de doctorat inédite). Université d'Ottawa, Ottawa, Ontario.

Noël, A. (1996). Présentation du numéro : vers un nouvel État-providence? Enjeux démocratiques. Politique et Sociétés, (30), 3-27. doi.org/10.7202/040032ar

Nunes, J. (2012). Health, Politics and Security, e-cadernos CES [Online], 15. doi: https://doi.org/10.4000/eces.989

Paul, P., et Ross-Kerr, J.C. (2011). « The Origins and Development of Nursing Education in Canada ». Dans J.C. Ross-Kerr et M.J. Wood, Canadian Nursing: Issues and Perspectives (5th ed) (pp.327-358). Mosby Canada.

Rafferty, A. M. (1996). The Politics of Nursing Knowledge. Routledge.

Rafferty, A. M., Robinson, J., et Elkan, R. (1997). Nursing history and the politics of welfare. Routledge.

Scott, F. R., Marsh, L., Spry, G., Gordon, J. K., Forsey, E., et Parkinson, J. S. (1975). Social planning for Canada. University of Toronto Press.

Therborn, G. (2011). «States, populations and productivity: towards a political theory of Welfare states ». Dans P. Lassman, Politics and social theory (Eds) (pp. 62-84). Routledge.

Ulmann, P. (2009). «La santé, facteur de croissance économique ». Dans : P.-L. Bras, Traité d'économie et de gestion de la santé (pp. 53-61). Presses de Sciences Po. 
Vivekanandan, B. (2002). Welfare state system in Canada: Emerging challenges. International Studies, 39(1), 45-63.

Vivekanandan, B. et Kurian, N. (2005). «Introduction: Welfare states and the future ». In: Vivekanandan, B. et Kurian, N. Welfare States and the Future (pp.1-14). Palgrave Macmillan.

Weir, G. M. (1932). Survey of nursing education in Canada. Toronto University Press.

Wood, V. (1984). Nursing Education: the move from hospital schools of nursing - an historical perspective. International Journal of Nursing Studies, 21(3), 183-192.

Wrede, S. (2010). « Nursing: Globalization of a Female-gendered Profession ». Dans E. Kuhlmann et E. Annandale, Gender and Healthcare (pp. 437-453). Palgrave Macmillan.

Wytenbroek, L., et Vandenberg, H. (2017). Reconsidérer l'histoire de la profession infirmière à l'occasion de Canada 150. L'infirmière Canadienne. https://infirmierecanadienne.com/fr/articles/issues/2017/juillet-aout-2017/reconsiderer-lhistoire-de-laprofession-infirmiere-a-loccasion-de-canada- 150 